ESJ Humanities

\title{
Caractérisation des déchets solides ménagers de Faladié
}

\author{
Oumar Bah
}

Docteur en Sciences de Gestion, Ecrivain - Relecteur - Enseignant -

Chercheur à l'Université Sup' Management, Mali

Boubacar Sanogo

Docteur en Sciences de Gestion,

Relecteur - Enseignant - Chercheur à l'Université de Ségou, Mali

Mohamed Traore

Docteur en Management Public, Enseignant - Chercheur à la FSEG

Doi:10.19044/esj.2021.v17n39p77

Submitted: 25 February 2021

Accepted: 10 November 2021

Published: 30 November 2021
Copyright 2021 Author(s)

Under Creative Commons BY-NC-ND

4.0 OPEN ACCESS

Cite As:

Bah O., Sanogo B. \& Traore M. (2021). Caractérisation des déchets solides ménagers de Faladié. European Scientific Journal, ESJ, 17 (39), 77.

https://doi.org/10.19044/esj.2021.v17n39p77

\section{Resumé}

Cet article est une étude expérimentale pour de connaitre la caractérisation et la quantification des déchets solides ménagers du quartier de Faladié dans la commune VI du District de Bamako. A Faladié, comme dans la plupart des communes de Bamako avec comme problématiques environnementales majeures, une insalubrité alarmante, qui accentue en partie les maladies comme : le paludisme, la fièvre typhoïde, le choléra, les infections respiratoires, les anémies, .... C'est dans ce contexte que la présente étude, voulant résorber l'épineuse problématique qu'est la gestion des ordures ménagères, a été coordonnée pour connaitre « la caractérisation des déchets ménagers au niveau des dépôts intermédiaires et finaux d'une part ; et de contribuer à la mise en place d'un système de tri adapté à Faladié d'autre part ». L'approche méthodologique d'échantillonnage utilisée pour déterminer la taille d'échantillon de déchets à trier est définie par la production journalière d'ordures ménagères d'un échantillon de la population totale par catégorie en satisfaisant à un niveau de confiance de $95 \%$. Ainsi, le tri manuel a porté sur le contenu de deux (2) bennes de $7 \mathrm{~m}^{3}$, un total de $14 \mathrm{~m}^{3}$ d'ordures ménagères, soit $7382,7 \mathrm{~kg}$. Dans ces conditions, l'étude a permis de dégager la caractérisation des ordures ménagères et de différencier les types de déchets 
valorisables de Faladié. En outre, nous avons déterminé qu'il est généré chaque jour $0,27 \mathrm{~kg}$ par habitant (soit $0,27 \mathrm{~kg} / \mathrm{habitant} / \mathrm{jour}$ ). A la suite de l'étude, des propositions stratégiques ont été formulées à la Mairie de la Commune VI dans le sens de mettre en place un comité de gestion intégrée des déchets.

Mots cles: Déchets solides ménagers, Trie des déchets, Caractérisation, Valorisation, Faladié

\title{
Characterization of Household Solid Waste in Faladie
}

\section{Oumar Bah}

Docteur en Sciences de Gestion, Ecrivain - Relecteur - Enseignant Chercheur à l'Université Sup' Management, Mali

Boubacar Sanogo

Docteur en Sciences de Gestion,

Relecteur - Enseignant - Chercheur à l'Université de Ségou, Mali

Mohamed Traore

Docteur en Management Public, Enseignant - Chercheur à la FSEG

\begin{abstract}
This article is an experimental study for the characterization and quantification of solid household waste in the Faladie neighborhood in the commune VI of the District of Bamako. In Faladie, as in most of the communes of Bamako with major environmental problems, an alarming insalubrity, which accentuates in part diseases such as: malaria, typhoid fever, cholera, respiratory infections, anaemia, It is in this context that the present study, aiming to solve the thorny problem of household waste management, was coordinated to find out « the characterization of household waste at the level of intermediate and final depots on the one hand; and to contribute to the setting up of an adapted sorting system in Faladie on the other hand». The methodological sampling approach used to determine the sample size of waste to be sorted is defined by the daily production of household waste of a sample of the total population by category at a $95 \%$ confidence level. Thus, the manual sorting involved the contents of two (2) $7 \mathrm{~m}^{3}$ skips, a total of $14 \mathrm{~m}^{3}$ of household waste, or 7382.7 kilograms. Under these conditions, the study allowed us to characterize the household waste and to differentiate between the types of recoverable waste in Faladie. In addition, we also determined that 0.27 kilogram per inhabitant is generated every day (i.e. 0.27 kilogram/inhabitant/day). Following the study, strategic proposals were formulated to the Commune VI Town Hall to set up an integrated waste
\end{abstract}


management committee.

Keywords: Household solid waste, Waste sorting, Characterization, Valorization, Faladie

\section{Introduction}

La gestion des déchets ménagers est un problème crucial dans les villes des pays en développement (PED) où les municipalités sont amenées à faire face à des quantités croissantes de déchets, avec des budgets très limités (NAQUIN. P., et NGNIKAM. E., 2008). L'accroissement de la production des déchets ménagers est lié à la forte croissance démographique et à l'urbanisation non maitrisée dans les pays du Sud (DIABATE. S., et al. 2005). Les récentes statistiques de la Banque Mondiale sur la production et les modes de gestion des déchets en Afrique Subsaharienne montrent que la situation de l'insalubrité urbaine tend à s'empirer. En effet, seulement $44 \%$, soit moins de la moitié des déchets produits sont collectés dans les municipalités de ces pays d'Afrique Subsaharienne, précise KAZA. S., et al. (2016). Au Mali, pour renforcer sa politique de gestion des déchets, l'Etat a revu le cadre juridique et institutionnel, transférant les compétences aux communes dans le cadre de la décentralisation. Cependant l'absence de transfert total des ressources en faveur des acteurs locaux fait plutôt croire à une décentralisation de la pénurie comme le souligne OTAYEK. R., (2009).

Ainsi, à la faveur de la politique de décentralisation malienne en février 1993, 703 communes urbaines et rurales ont été créés au Mali. Ces communes sont administrées de façon institutionnelle avec les autres structures des collectivités. Renforcée par la loi du 12 avril 1995 portant code des collectivités territoriales, la politique de la décentralisation a ouvert la voie à une plus grande responsabilisation des collectivités locales. Désormais, elles sont responsables de leur propre développement et peuvent confier à toute structure ou organisation privée de leur choix (indépendant, entreprise, GIE, association, coopérative, etc.) le soin d'assurer à leur place un service public. C'est le cas, entre autres, de l'assainissement dans la Commune VI de Bamako à travers le GIE - DJIGUI depuis 1992.

En matière de gestion des déchets solides, les communes du district de Bamako ont la charge d'assurer la pré collecte, et de l'acheminer depuis les ménages vers le dépôt de transit. Cette tâche est confiée au secteur privé (GIE, coopératives). Le district de Bamako a également en charge la collecte secondaire (et l'acheminement des déchets depuis les ménages vers les dépôts de transit) par le biais de la Direction des Services Urbains de Voirie et d'Assainissement (DSUVA), qui est affaiblie par un manque de matériel approprié et d'une décharge finale à Bamako. Pour cette raison, les dépôts 
intermédiaires finissent par se transformer en dépôts finaux (rapport du GIE Faso Kanu, 1992).

L'objectif de cette étude est de caractériser les déchets solides ménagers de Faladié afin de connaître leur composition et de proposer une méthode de valorisation des déchets.

\section{Matériel et méthodes}

Site de l'étude : Le site de cette étude est le centre de compostage de Faladié qui a été mis à notre disposition pour les besoins de l'étude. Aménagé par la Mairie de la Commune VI pour abriter les études et expositions sur les déchets et études sur l'environnement. Ce centre a été mis à notre disposition pour les besoins de l'étude.

Le quartier de Faladié relevant du centre secondaire d'Etat Civil de Banankabougou - Faladié, situé dans la commune VI du district de Bamako, Mali.

La Commune VI couvre la partie Sud - Est du District de Bamako sur la rive droite du fleuve Niger et s'étend sur $94 \mathrm{~km}^{2}$. Elle compte 76436 ménages (suivant le Recensement Général de la Population et de l'Habitat du Mali de 2009 (RGPH 2009)) qui ont un niveau de revenu ne permettant pas d'assurer une vie décente.

La Commune VI comprend 10 quartiers dont 3 à caractère urbain (Faladié, Banankabougou, Sogoniko) et 7 semi - rural (Dianéguéla, Missabougou, Niamakoro, Sokorodji, Sénou et Magnambougou et Yirimadio) (selon le Rapport provisoire SNV : "Répertoire des GIE de pré collecte d'ordures ménagers intervenant dans le District de Bamako: cas de la commune VI», étude diagnostique, août 2008).

Faladié a une population de 68568 habitants dont 11651 ménages en 2009, avec une superficie de 744 hectares (suivant le Recensement Général de la Population et de l'Habitat du Mali de 2009 (RGPH 2009)). Le quartier est divisé en cinq (5) secteurs qui sont : Faladié Socoura, Faladié Socoro, Faladié Solola, Faladié Carrefour et Faladié Sema. 


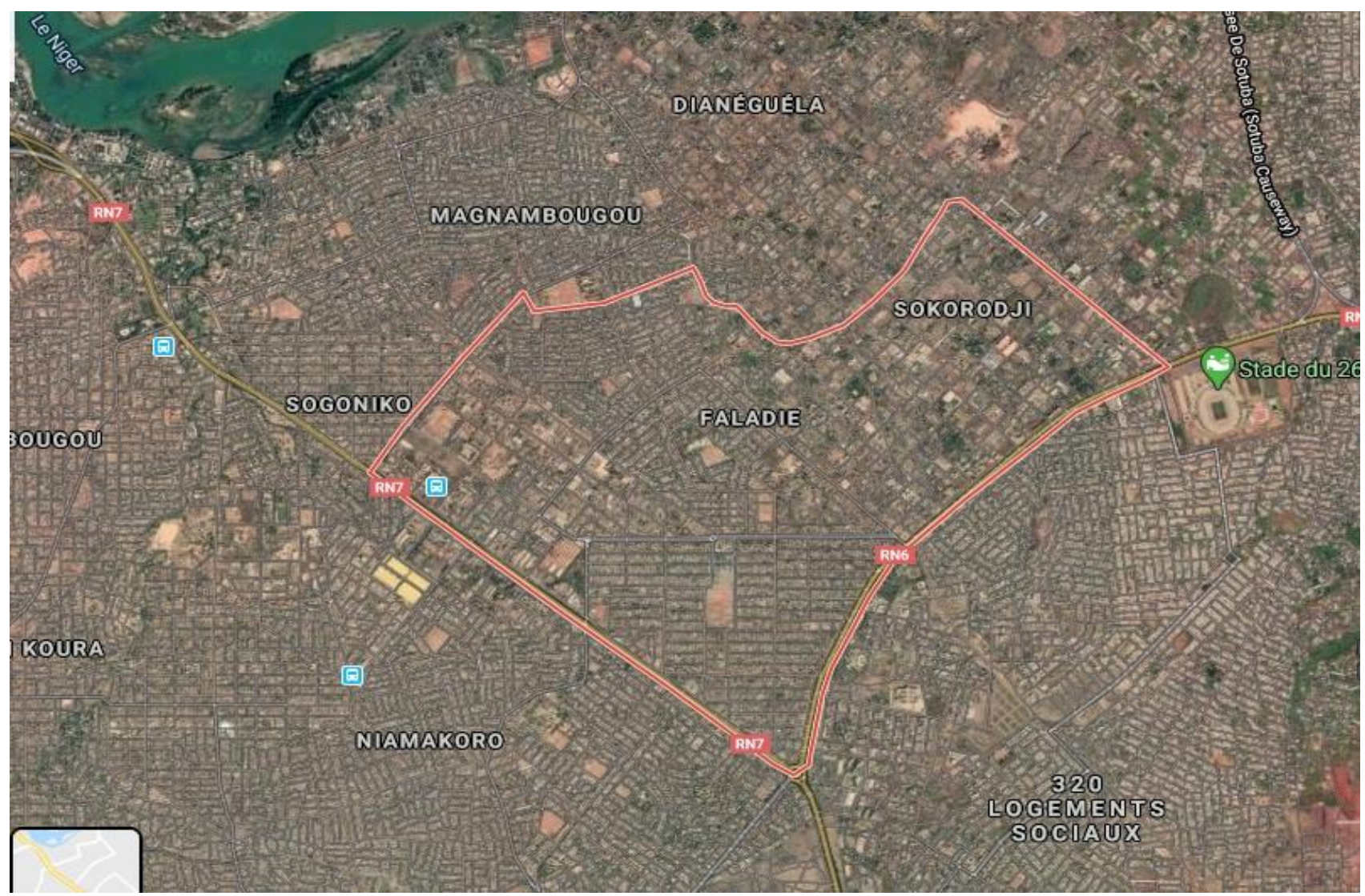

Figure 1 : Carte de localisation par satellite de Faladié en commune VI de Bamako

Source : La carte satellitaire de Faladié, la zone de collecte des déchets et abritant le site du centre de collecte provient du site :

https://www.google.com/maps/place/Faladie,+Bamako/@12.6002204,-

$7.9621881,5961 \mathrm{~m} / \mathrm{data}=! 3 \mathrm{~m} 1$ !1e3!4m5!3m4!1s0xe51d21eb9175d4d:0xd5aa68af705d3819!

$8 \mathrm{~m} 2 ! 3 \mathrm{~d} 12.6039148 ! 4 \mathrm{~d}-7.9456687$

\section{Méthodes}

Dans le cadre des préparatifs de cette activité, nous avons réalisé quelques visites (la visite à la mairie de Faladiè - Banankabougou, à Sema Saniya et au GIE SADE) qui nous a permis de connaître la répartition géographique sur le plan Assainissement ainsi que le volume de déchets ménagers produit par chaque GIE et par jour. Malheureusement aucun des GIE n'a pu nous fournir la composition des déchets ménagers qu'ils ramassent. Ce qui explique l'orientation de cette recherche sur la caractérisation et la quantification des déchets ménagers.

Une expérimentation de tri portant sur une période de deux semaines au centre de compostage de Faladiè a été diligentée. L'activité consistait à trier manuellement le contenu de 2 bennes de $7 \mathrm{~m}^{3}$ soit $14 \mathrm{~m}^{3}$ au total. Cette expérimentation nous a permis de déterminer non seulement la quantité de déchets solides collectés par le GIE SADE sur une journée de ramassage ; mais 
également à partir du tri multiple de mettre en exergue les différents types de déchets valorisables, le contenu des $14 \mathrm{~m}^{3}$ de déchets ménagers organiques.

\section{Le Comité de Gestion et de Valorisation des Déchets (COGEVAD)}

Le COGEVAD est une plateforme communale initiée en 2000 par la société civile en Commune VI du District de Bamako (Rapport annuel du COGEVAD, décembre 2009).

Le COGEVAD, est composé des structures suivantes : la Mairie de la Commune VI, les services techniques ayant des activités directement liées à l'assainissement au niveau de la mairie (SACPN, BH, BUPE, DSUVA), les GIE intervenant dans la collecte des ordures ménagères, les comités de salubrité, la CAFO, la Coordination Nationale des Jeunes (CNJ) en Commune VI et les maraîchers. Le COGEVAD bénéficie de l'appui de plusieurs partenaires maliens et étrangers tels que le CEK Kala Saba, la GIZ Entwicklungsdienst, la SNV, WaterAid et APIDEC.

En commune VI, il existe un dépôt de transit officiel à Magnambougou connu sous le nom de dépôt de Dianéguéla (démantelé actuellement) d'une part, et plusieurs dépôt anarchique (espace non prévu à cet effet à savoir : zone ATTbougou, ancienne carrière de Sirakoro, Bollé, zone aéroportuaire, champs) d'autre part.

Compte tenu de nos moyens financiers, l'étude s'est focalisée sur le quartier de Faladié relevant du centre secondaire d'Etat Civil de Banankabougou - Faladié.

Faladiè a une population de 68568 habitants dont 11651 ménages en 2009, avec une superficie de $\mathbf{7 4 4}$ hectares (suivant dernier Recensement Général de la Population et de l'Habitat du Mali de 2009 (RGPH 2009)).

Le quartier est divisé en cinq secteurs qui sont :

- Faladié Socoura ;

- Faladié Socoro ;

- Faladié Solola ;

- Faladié Carrefour et

- Faladié Sema.

Il existe deux grands GIE à Faladiè à savoir : SADE et Sema Saniya. Le GIE Sema Saniya évolue précisément à Faladié Sema, Faladié Solola, Bolé et Faladié Socoro. Il compte environ 2500 abonnés environ et dispose d'un camion, de trois tracteurs moteurs comme parc automobile.

Le GIE SADE quant à lui évolue à Faladié Socoura et Faladié carrefour avec près de 750 abonnés environ; et dispose d'un camion benne.

Le GIE SADE produit au total $21 \mathrm{~m}^{3}$ de déchets ménagers organiques par jour. Ainsi, la quantité de déchets contenue dans $21 \mathrm{~m}^{3}$ est estimée à 11 074,05 kg. 
La production de déchets du GIE SADE est estimée à 11 074,05 kg par jour soit une estimation mensuelle de $310073,4 \mathrm{~kg}$ pour le mois de Février, ce qui correspond à $588 \mathrm{~m}^{3}$.

NB : Les résultats obtenus de cette étude concernent la zone couverte par le GIE SADE.

\section{Résultats de l'expérimentation}

Les résultats ont été obtenus à l'issus d'un premier tri au centre de compostage de Faladié. Ils seront présentés suivant deux optiques, à savoir : l'optique de la caractérisation et celle de la valorisation.

\subsection{Caractérisation et quantification des déchets ménagers}

Les résultats figurent sur le tableau 1, il dresse la caractérisation des déchets ménagers produits par les secteurs couverts par le GIE SADE. La quantité de déchets contenue dans $14 \mathrm{~m}^{3}$ est évaluée $7382,7 \mathrm{Kg}$ (en poids, deux semaines après la collecte).

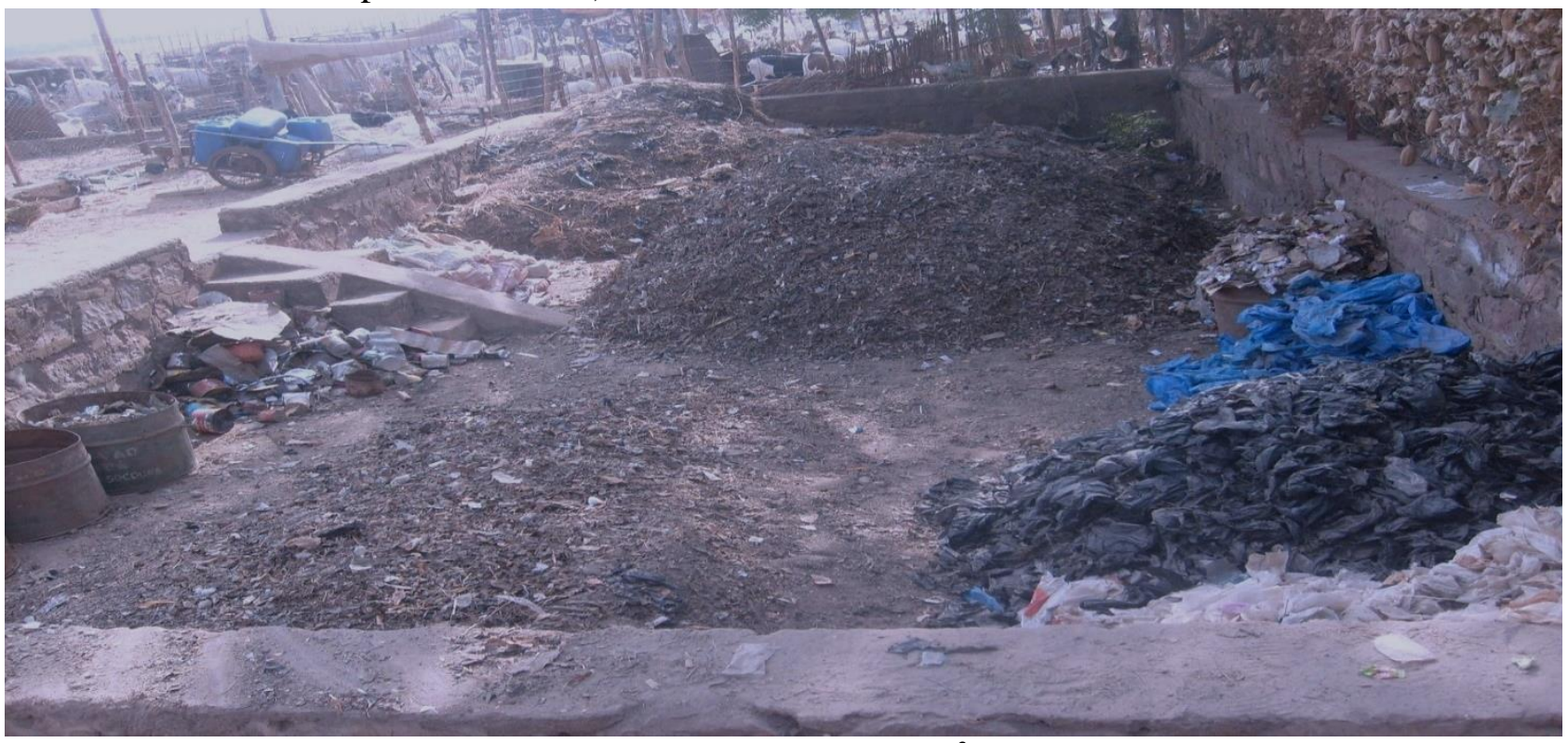

Photo 1 : Photo des déchets ménagers de $14 \mathbf{~ m}^{3}$ après le premier tri

Source : Etude d'expérimentation, (Février 2011), photo prise lors l'expérimentation. 
Tableau 1 : Caractérisation des déchets des $14 \mathrm{~m}^{3}(7382,7 \mathrm{Kg})$

\begin{tabular}{|l|l|l|}
\hline Eléments & Quantités en kg & Poids en \% \\
\hline Déchets de cuisines & 323,4 & 4,4 \\
\hline Reste d'aliments & 263,4 & \\
Légumes et fruits & 60 & \\
\hline Matières Plastiques & 141,1 & 1,9 \\
\hline PVC en bouteilles & 114,5 & \\
Sacs vides & 12 & \\
Mèches & 10 & \\
Chiffon & 4,6 & \\
\hline Sachets en plastiques & 380,2 & 5,1 \\
\hline Sachets blancs & 144 & \\
Sachets noirs & 178 & \\
Sachets bleus & 6,2 & \\
Sachets divers & 52 & 0,6 \\
\hline Chaussures et caoutchoucs & 41,8 & 2,6 \\
\hline Textiles & 192 & \\
\hline Habits & 157 & \\
Couches enfants & 35 & 2,5 \\
\hline Papiers & 187,7 & 0,6 \\
\hline Déchets d'animaux & 41,8 & \\
\hline Cornes d'animaux & 1,3 & \\
Os d'animaux & 39 & 53,1 \\
Plumes, poils et peau & 1,5 & 3,9 \\
\hline Mélange de sable, terre et cendre & 3920 & 0,6 \\
\hline Cailloux & 287 & 0,7 \\
\hline Verre et céramique & 41,4 & \\
\hline Métaux (bronze, fer, aluminium et zinc) & 53,3 & \\
\hline Déchets végétaux & 1752,2 & \\
\hline Feuilles & 1642,5 & \\
Bois & 90,7 & \\
Charbon & 19 & \\
\hline Déchets hospitaliers & 8 & \\
\hline Déchets toxiques & 12,8 & \\
\hline Piles et batteries téléphones & 12 & \\
Ampoules & 0,8 & \\
\hline TOTAL & $\mathbf{7 3 8 2 , 7}$ & \\
\hline & & \\
\hline
\end{tabular}

Source : Etude d'expérimentation, (Février 2011).

Les ordures ménagères sont principalement composées de mélange (terre, sable et cendre), 53,1\%, de déchets végétaux à hauteur de $23,7 \%$. Le reste regroupant les déchets de cuisines, de matières plastiques, de sachets, de textiles, de papiers, de déchets d'animaux, de cailloux, de verre et céramique, de métaux, de déchets hospitaliers et de déchets toxiques de $23,2 \%$. 


\subsection{Déchets valorisables et non valorisables}

Le tableau ci-après reprend la caractérisation des déchets ménagers, en les classant par types de transformations ou réutilisations possibles (caractère valorisable ou non).

Tableau 2 : Déchets valorisables et non valorisables

\begin{tabular}{|l|l|l|}
\hline Eléments & $\begin{array}{l}\text { Quantités } \\
\text { kg }\end{array}$ & $\begin{array}{l}\text { Poids en } \\
\text { \% }\end{array}$ \\
\hline Déchets dégradables / compostages (Valorisables) & 2437,8 & 33 \\
\hline Reste d'aliments & 263,4 & \\
Légumes et fruits & 60 & \\
Feuilles & 1642,5 & \\
Bois & 90,7 & \\
Papiers (non souillés) & 187,7 & \\
Textiles & 192 & \\
Résidus de peau & 1,5 & 9 \\
\hline Déchets non dégradables / recyclages (Valorisables) & 659,1 & \\
\hline Métaux & 53,3 & \\
PVC en bouteilles & 114,5 & \\
Sacs vides & 12 & \\
Mèches & 10 & \\
Chiffon & 4,6 & \\
Sachets & 380,2 & \\
Verre et céramique & 41,4 & \\
Chaussures et caoutchoucs & 41,8 & \\
Cornes d'animaux & 1,3 & \\
\hline Déchets non dégradables et toxiques (non valorisables) & 20,8 & \\
\hline Déchets hospitaliers & 8 & \\
Piles et ampoules (plomb) & 12,8 & \\
\hline Inertes (non valorisables mais réutilisables) & 4265 & \\
\hline Mélange de sable, terre et cendre & 3920 & 57,7 \\
Cailloux & 287 & \\
Charbon & 19 & \\
Os d'animaux & 39 & \\
\hline TOTAL & $\mathbf{7 3 8 2 , 7}$ & $\mathbf{1 0 0}$ \\
\hline
\end{tabular}

Source : Etude d'expérimentation, (Février 2011).

Les ordures ménagères sont étudiées pour mettre en évidence les différentes transformations ou réutilisations possibles.

Déchets valorisables : La principale estimation relative à la fraction biodégradable :

$\mathbf{2 4 3 7 , 8} \mathbf{~ k g}$ de déchets dégradables ont été collectés (33\% du poids total). Il s'agit des matières qui peuvent être dégradées par l'action des micro-organismes en un laps de temps déterminé : reste d'aliments, légumes et fruits, feuilles, bois, papiers non souillés, textiles biodégradables et résidus de peau. 
Au Mali le compostage relève de la compétence des GIE et des particuliers qui n'utilisent pas des déchets de cuisine, de feuilles. Les composts utilisés dans les champs proviennent des dépôts de transit ou sont directement transportés des ménages aux champs.

La principale estimation et conclusion relatives à la fraction recyclable :

> $\mathbf{6 5 9 , 1} \mathrm{kg}$ de déchets recyclables ont été triés ( $9 \%$ du poids total). Ce sont des matières qui ne peuvent pas être dégradées par l'action des micro-organismes en un laps de temps déterminé : métaux, $\mathrm{PVC}$ en bouteilles, sacs vides, mèches, chiffons, sachets, verre et céramique, chaussures et caoutchoucs et cornes d'animaux.

Les ordures valorisées se négocient soit sur place soit revendues à d'autres utilisateurs au Mali ou à l'extérieur du Mali. Les différentes utilisations sont :

$>\mathrm{La}$ ferraille (fer, aluminium, zinc, bronze), elle est vendue aux ferrailleurs au prix du marché et exportée à l'extérieur du Mali pour la transformation ;

$>$ Les canettes de boisson et boites de conserve sont utilisés pour en faire des jouets (avion, voiture etc.) ;

Les PVC en bouteilles sont lavés et vendus au Mali à des vendeurs de jus, de médicaments traditionnels etc.;

$>$ Les sacs vides sont transformés sur place en corde par les cordonniers ;

$>$ Les chaussures usées et seaux plastiques sont vendus et transformés sur place ou à l'extérieur ;

$>$ Les sachets sont triés selon la qualité et vendus à des unités de transformations pour en faire des gaines, d'articles en plastiques.

$>$ Les cornes d'animaux sont exportées et utilisées à d'autres fins (flûtes, ornement de maison etc.).

Il convient de mentionner que dans la cour de la Fédération Nationale des Artisans du Mali (FNAM) se trouve des associations féminines qui transforment des déchets plastiques pour en faire des habits par le tissage, des paniers, des sacs à main et des chaises.

Déchets non valorisables : La principale estimation et conclusion relatives à la fraction déchets toxiques :

$\mathbf{2 0 , 8} \mathbf{~ k g}$ de déchets toxiques ont été récupérés ( $0,3 \%$ du poids total), ce sont des matières qui relâchent des contaminants chimiques dans le sol : déchets hospitaliers, piles et ampoules (plomb).

Exemples : «Une pile à bouton abandonnée dans la nature pollue $1 m^{3}$ de terre ou 400 litres d'eau pendant 50 ans » (IBGE - BIM (2004)). 
« Le plomb, accumulé dans la chaîne alimentaire est responsable chez l'homme: des troubles respiratoires; de lésions du foie; de troubles de l'ossification; d'anémies; de lésions du système nerveux ». (CAZALAS 1993).

Déchets réutilisables : La principale estimation relative à la fraction inerte :

$>4265 \mathrm{~kg}$ de déchets inertes ont été collectés (soit 57,7\% du poids total), ce sont des matières sans vie mais peuvent être réutilisés dans la vie quotidienne, tels que l'utilisation du mélange de sable, terre et cendre, cailloux et les os d'animaux dans la construction ou le remplissage des anciennes carrières, la réutilisation du charbon.

Cette fraction présente des disparités géographiques importantes : elle est plus élevée dans les localités à faibles revenus.

Quelques photos des déchets ménagers après tri (reparties en valorisables à gauche et non valorisables à droite)
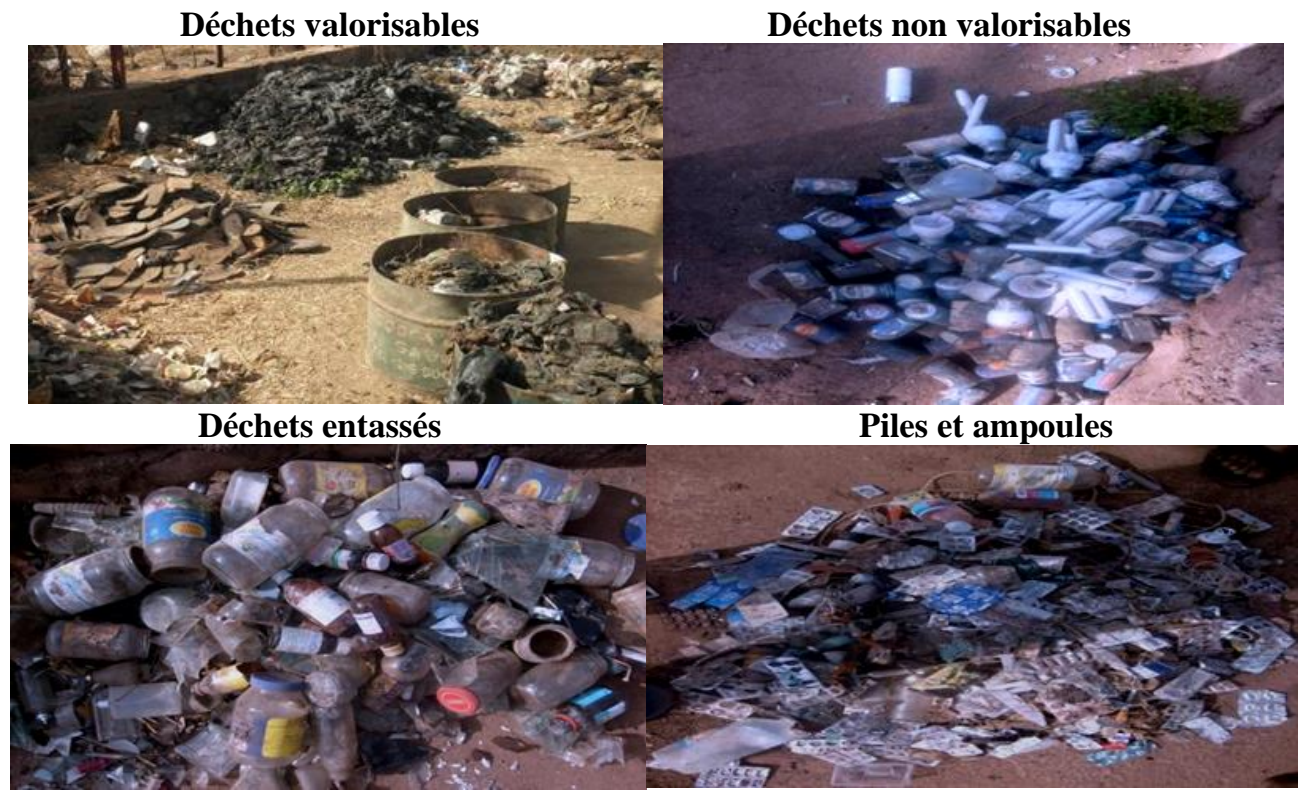

Verres

Produits pharmaceutiques

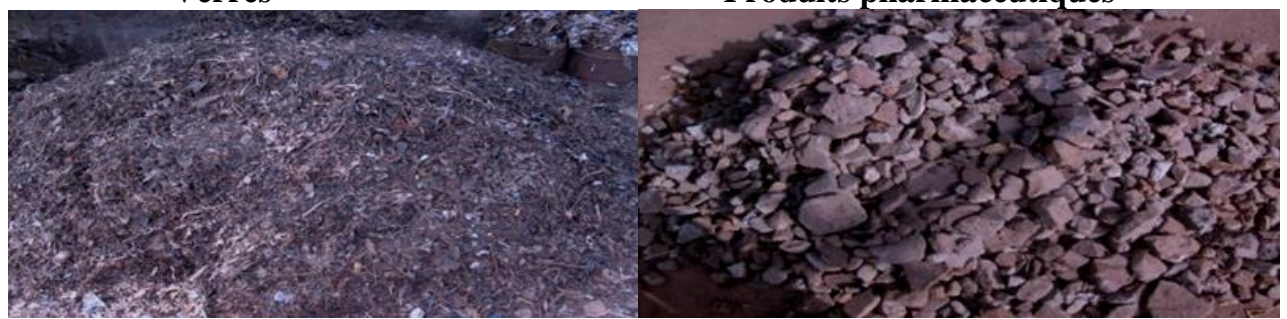

Source : Etude d'expérimentation, (Février 2011), Photos prisent lors l'expérimentation. 


\section{Discussion des résultats}

La caractérisation des déchets ménagers de Faladié à l'issus d'un premier tri au centre de compostage de Faladié à donner des proportions principalement composées de mélange (terre, sable et cendre), 53,1\%, de déchets végétaux à hauteur de $23,7 \%$. Le reste regroupant les déchets de cuisines, de matières plastiques, de sachets, de textiles, de papiers, de déchets d'animaux, de cailloux, de verre et céramique, de métaux, de déchets hospitaliers et de déchets toxiques de 23,2\%.

Sur la caractérisation des déchets ménagers et la contamination du sol, nous avons les travaux d'ADJIRI. O., et al. (2008) en Côte d'ivoire qui aborde dans le même sens avec des proportions légèrement différentes. Il en est de même dans la thèse de doctorat d'ALOUEIMINE. S. O., (2006) en Mauritanie. A la différence que ce dernier à réaliser une étude proposant une méthodologie de caractérisation des déchets ménagers adaptée à la ville de Nouakchott. Son travail à contribuer la proposition d'outils d'aide à la décision et une gestion stratégique des déchets. Dans le même sens, BEN AMMAR S., (octobre 2006) en Tunisie, a mis au point une méthode adaptée à la gestion des déchets ménagers. MAQUERON. G., (25 Novembre 2009), dans son dossier pense que : la gestion, le traitement et le recyclage des déchets découlent d'un constat : les volumes de déchets se sont multipliés depuis 1950, ainsi que leurs effets nocifs pour la santé, l'environnement et l'économie. La gestion des déchets est une étape cruciale. Si l'on se contentait d'entreposer ses déchets dans des décharges, le problème de leur accumulation se poserait (et se pose) car, suivant leur nature, ils peuvent se dégrader, et donc disparaître, très lentement. D'autres auteurs pensent par rapport à ce point que les déchets d'équipements électriques et électroniques, les pneumatiques, les matières plastiques et des déchets médicaux présents sur les décharges sauvages de déchets ménagers peuvent être sources d'une forte concentration des métaux lourds dans les sols (BOUCHAKOR, MALAYERI cités par BODJONA et al. 2012). La caractérisation des matières organiques dans les matrices déchets en vue de proposer des indicateurs de dégradation de la qualité des eaux a été réalisée par SEGBEAYA. N. K., et al. (2012). Sur les aspects de contamination du sol, nous citerons le cas de BODJONA, (2012) qui a étudié l'impact de quelques métaux lourds (plomb, cadmium, nickel, cuivre et du zinc) sur la contamination du sol d'une décharge finale au Togo. KJELDSEN. P., et al. (2002) ont travaillé sur la composition actuelle et à long terme des lixiviats des décharges. KOUAME. K. I., (2007) à étudier dans sa thèse de doctorat le degré de pollution physico - chimique et proposa une analyse du risque de contamination de la nappe par un modèle de simulation des écoulements et du transport des polluants à Akouédo, en Côte d'Ivoire. KOUADIO. G., et al. (2000); ont dans leur étude mit l'accent sur la 
détermination de la pollution chimique des eaux de la zone de la décharge d'Akouédo, en Côte d'Ivoire.

Sur la filière déchet proprement dit et la valorisation des déchets, nous pouvons citer les travaux réalisés par le COGEVAD (décembre 2009) au Mali, à travers leur rapport annuel qui donne des stratégies pour la valorisation des déchets ménagers par la production de composte organique. Association Jeunesse Entreprenariat, (Février 2011) a travaillé à travers leur première étude sur l'environnement sur les déchets ménagers de Faladié à travers le GIE SADE (qui s'occupe du ramassage d'ordure dans le quartier). KOLEDZI. K. E., et al. (2011a) et (2011b) proposent des techniques de compostage et la valorisation de l'agriculture à travers le compostage à Lomé. TCHEGUENI. S., (2011) à travers une étude propose une technique de valorisation des déchets agroalimentaire au Togo.

Pour ce qui concerne les études réalisés sur la filière déchets proprement dit, nous pouvons citer les travaux du GIE JIGUI (Octobre 1992) au Mali sur la gestion des déchets à travers l'expérience du projet « Besseya ». Nous pouvons également citer le cas du GIE Faso Kanu (1992) qui proposa également une technique de gestion des déchets à travers le projet «KANA » à Bamako. La gestion des déchets à travers les régis de quartiers a également fait l'objet d'une étude par BALI. A., (juin 1993) au Mali. L'élimination des déchets solides en tenant compte des aspects environnementaux a été développée par CHALABI. M., et al. (2001) au Maroc. La gestion d'une décharge publique à travers la caractérisation des lixiviats pour connaitre leur impact sur les ressources en eau a été étudiée par HAKKOU. R., (2001) au Maroc. HARPET. C., (2003), quant à lui, s'est intéressé à l'anthropologie des décharges et l'évaluation interdisciplinaire des risques sanitaires. DIABAGATE. S., et al. (2005) proposèrent un guide pratique sur la gestion des déchets ménagers et des sites d'enfouissements à travers les techniques utilisées dans les pays du sud. Au Maroc, l'étude d'impact d'une décharge a fait l'objet d'une étude par EL KHAMLICHI. M. A., et al. (1997). KOLEDZI. E., et al. (Mars 2015), ont mis en exergue les problématiques de la gestion des déchets dans les villes en développement à travers le transfert des déchets, l'adaptation du schéma et les sources de financement. KAPEPULA. L., et al. (12 janvier 2016) proposèrent un modèle thermogravimétrique d'évaluation des rendements de pyrolyse et de gazéification de la fraction fermentescible des déchets ménagers à Bujumbura. KAPEPULA. L., et al. (juillet 2016) proposèrent un état des lieux de déchets solides ménagers dans la ville D’Uvira, Sud - Kivu, République Démocratique du Congo. NKITUAHANGA YENAMAU. A., (2009) pense que la gestion des ordures ménagères telles pratiquées dans la commune de Masina ne contribue pas au développement durable. Autrement les ordures ménagères ne suivent pas la filière normale d'une gestion durable. NTABUGI KIKONGO M. M., (21 août 2013), à partir 
des résultats obtenus dans son étude, affirme que la population de la ville de Bukavu et particulièrement celle de la commune d'Ibanda n'a pas des connaissances suffisantes sur la gestion des déchets ménagers. Cette lacune pourrait exposer la population et la biodiversité aux maladies à travers les infections par les microbes et d'autres polluants liés à ces déchets. MOKHTARIA. M. M., et al. (2007) dans leur étude ont travaillé sur les caractéristiques de la décharge publique de la ville de Tiaret et son impact sur la qualité des eaux souterraines. La pollution par les déchets et l'impact des déchets sur l'environnement et la santé furent développés par NGO. C., et al. (2004) dans leur étude.

\section{Conclusion}

Les résultats obtenus dans la présente étude permettent de de connaitre la caractérisation et la quantification des déchets ménagers de Faladié. Ils nous ont permis de proposer des stratégies pour une gestion rationnelle des déchets ménagers d'une part; et de proposer des solutions qui sous - tendent la valorisation et le recyclage. Il convient de préciser que nos hypothèses ont été vérifiées. Tout au long de cette étude, nous avons mis en exergue que les déchets organiques peuvent être trié, et le résultat du tri est soit réutilisable soit valorisable.

Par ailleurs, il est nécessaire de remarquer que Faladié n'a jamais fait l'objet d'une étude de caractérisation et de valorisation des déchets ménagers publiée. Au regard des résultats obtenus, il est nécessaire de dupliquer ces genres d'études sur les autres décharges de la Commune VI du district de Bamako, afin de mieux cerner la composition des déchets dans l'optique d'une valorisation. Nous pouvons donc affirmer qu'en gérant bien nos ordures ménagères, nous pouvons "créer de la valeur ajouté », cela engendrerait des créations d'emploi et d'autres types d'Activités Génératrices de Revenu (AGR) autour de filière des déchets ménagers.

\section{References:}

1. ADJIRI. O. A., GONE. D. L., KOUAME. I. K., KAMAGATE. B., BIEMI. J., (2008). "Caractérisation de la pollution chimique et bactériologique de l'environnement de la décharge d'Akouedo Abidjan-Côte d'Ivoire », International journal of Biological and Chemical Scientific, Vol. 2, n $4,401-410$.

2. ALOUEIMINE. S. O., (2006); « Méthodologie de caractérisation des déchets ménagers à Nouakchott (Mauritanie) : contribution à la gestion des déchets et outils d'aide à la décision "; Thèse de doctorat $\mathrm{N}^{\circ} 012$ en Chimie et microbiologie de l'eau Soutenue à Limoges, en partenariat avec Université de Limoges. Faculté des sciences et techniques (autre partenaire) . Sous la direction de Guy MATEJK. 
3. Association Jeunesse Entreprenariat (J\&E), (Février 2011), « Première étude sur l'analyse des déchets ménagers de Faladié en Commune VI de Bamako: cas du GIE SADE», Récépissé de déclaration d'association $n^{\circ} 639$ - G - DB, Siège social : Banankabougou Sema Rue : 611, Porte : 392 Bamako / Mali, Tél : (223) 76208524 / (223)

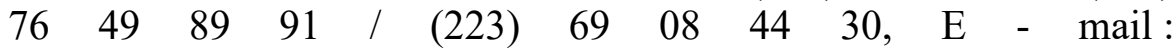
jeunesseentreprenariat@gmail.com.

4. Association Jeunesse Entreprenariat (J\&E), (Septembre 2011), "Projet expérimental de valorisation des déchets organiques ménagers par la production de compost biologique à destination de l'agriculture péri urbaine ", Récépissé de déclaration d'association $\mathrm{n}^{\circ} 639$ - G - DB, Siège social : Banankabougou Sema Rue : 611, Porte : 392 Bamako / Mali, Tél : (223) 76208524 / (223) 76498991 / (223) 690844 30, E - mail : jeunesseentreprenariat@gmail.com.

5. BALI. A., (juin 1993); "Mission "Filière déchets urbains" à Bamako (Mali), Comité national de liaison des régies de quartier $(C N L R Q) » ; 31$ pages.

6. Banque Mondiale., (2014); «Results-based Financing for Municipal Solid Waste. Knowledge papers ». Urban Development Series. World Bank, avec la participation de: KAZA. S., YAO. L. C., \& STOWELL. A.

7. Banque Mondiale., (2016); «World Bank Paper: Sustainable Financing and Policy Models for Municipal Composting. Knowledge papers ». Urban Development Series. World Bank, avec la participation de : KAZA. S., YAO. L. C., \& STOWELL. A.

8. Banque Mondiale., (2018); "What a Waste 2.0: A Global Snapshot of Solid Waste Management to $2050 »$. Urban Development; Washington, DC: World Bank. World Bank, avec la participation de: KAZA. S., YAO. L. C., BHADA - TATA. P., \& VAN WOERDEN. F. https://openknowledge.worldbank.org/handle/10986/30317.

9. BECHAC. J. P., BOUTIN. P., NUER. P., (1984); «Traitement des eaux usées ». Ed. Eyrolls. Paris.

10. BEN AMMAR. S., (octobre 2006); "Les enjeux de la caractérisation des déchets ménagers pour le choix des traitements adaptés dans les pays en développement : Résultats de la caractérisation dans le grand Tunis, Mise au point d'une méthode adaptée ». Thèse de doctorat de l'Institut National Polytechnique de Lorraine.

11. BODJONA., (2012); "Contamination du sol de la décharge finale de la ville de Lomé par les métaux lourds : Cas du plomb, cadmium, nickel, cuivre et du zinc». Thèse de doctorat $\mathrm{N}^{\circ}$ 455/FDS de l’Université de Lomé. 
12. CAZALAS. F., (1993) ; «Manuel d'initiation à l'environnement», Fondation Ushuaia, les éditions de L'environnement, page 109.

13. CHALABI. M., BENMBAREK. M., MAGAA. K., (2001) ; «Rapport sur l'élimination des déchets solides de la ville de Meknès, aspects environnementaux». Royaume du Maroc, Ministère de l'Aménagement du Territoire, de l'Urbanisme, de l'Habitat et de l'Environnement. Département de l'environnement secrétariat général.

14. Comité de Gestion et de Valorisation des Déchets (COGEVAD), (décembre 2009), «Rapport annuel ».

15. DIABAGATE. S., (2007) ; «Assainissement et Gestion des ordures ménagères à Abobo, cas d'Abobo - Baoule »; Mémoire de Master, Institut de Géographie Tropicale / Université d'Abidjan, 96 pages.

16. DIABAGATE. S., et THONART. P., HILIGSMANN. S., \& LARDINOIS. M., (2005) ; "Guide pratique sur la gestion des déchets ménagers et des sites d'enfouissements techniques dans les pays du $s u d »$, collection points de repère. Retrieved from ww.iepf.org.

17. EL KHAMLICHI. M. A., LAKRABNI. S., KABBAJ. M., JARBY. E., KOUHEN. M., (1997); "Etude d'impact de la décharge d'Akrach (Rabat, Maroc) sur la qualité des ressources en eau», Revue Marocaine Civile, ${ }^{\circ} 68,17-31$.

18. ESSAHLAOUI. A., (2000); «Contribution à la reconnaissance des formations aquifères dans le bassin de Meknès- Fès (Maroc) ». Thèse de Doctorat, Ecole Mohammedia des ingénieurs, Rabat, Maroc.

19. GIE Faso Kanu., (1992) ; «Etude de faisabilité du projet "KANA », Bamako (Mali)», GIE Faso Kanu, 13 pages.

20. GIE JIGUI., (Octobre 1992); "L'expérience du projet BESEYA, Bamako (Mali)», GIE JIGUI, 11 pages.

21. HAKKOU. R., (2001); "La décharge publique de Marrakech : caractérisation des lixiviats, étude de leur impact sur les ressources en eau et essai de leur traitement ». Thèse de doctorat d'Etat, Université de CADDI AYYAD, Maroc, 150 pages.

22. HAKKOU. R., WAHBI. M., BACHNOU. A., ELAMARI. K., HANICH., HIBTI. M., (2001); "Impact de la décharge publique de Marrakech (Maroc) sur les ressources en eau », Bull. Eng. Geol. Env., 60, 325-336.

23. HARPET. C., (2003); «De l'anthropologie des décharges à l'évaluation interdisciplinaire des risques sanitaires ». Natures, Sciences, Sociétés 11 (2003) 361-370. Doi : 10.1016/S12401307(03)00116-X.

24. Institut National de la Statistique (INSTAT). (2009) ; «Recensement Général de la Population et de l'Habitat du Mali (RGPH)». 
25. Institut Bruxellois pour la Gestion de l'Environnement (IBGE - BIM) : (2004) ; «Comment éviter les déchets dangereux?». Coordination : P. De Mulder (IBGE) avec le soutien de l'Observatoire Bruxellois de la Consommation Durable (OBCD) ; Comité de lecture : C. Bouland, L. Demanet, R. De Laet et J. Van Bambeke (IBGE) ; C. Rousseau (OBCD). Illustrations : L. Poma (Studio Poca) Graphisme \& Design : L. Defaweux et M. Desager (IBGE) Ed. Resp. : J. P. Hannequart et E. Schamp, Gulledelle, 100 - 1200 Bruxelles Dépôt légal : D/5762/2004/21 Imprimé à l'encre végétale sur papier recyclé.

26. KANE. B., (Août 2004) ; " monographie de la commune VI du district de Bamako », Mairie de la Commune VI (SACPN).

27. KAPEPULA. L., MUYISA. S., et JUNG. G. C., (juillet 2016); "Contribution à l'état des lieux de déchets solides ménagers dans la ville D’Uvira, Sud-Kivu, République Démocratique du Congo». Article publié à International Journal of Biological and chemical sciences DOI: 10.4314/ijbcs.v10i3.39. e-ISSN: 1997-342X. print ISSN: 1991-8631.

28. KAPEPULA. L., NDIKUMANA. T., et JUNG. G. C., (12 janvier 2016) ; "Modèle thermogravimétrique d'évaluation des rendements de pyrolyse et de gazéification de la fraction fermentescible des déchets ménagers à Bujumbura»; Article publié à Afrique science. Reference: http://www.afriquescience.info. ISSN: 1813-548X.

29. KJELDSEN. P., BARLAZ. M. A., ROOKER. A. P., BAUM A., LEDIN. A., CHRISTENSEN. T. A., (2002); «Present and long-term composition of MSW landfill leachate », Crit. Rev., Environ. Sci. Technol., Vol. 32, n 4 , 297-336.

30. KOUAME. K. I., (2007) ; «Pollution physico-chimique des eaux dans la zone de la décharge d'Akouedo et analyse du risque de contamination de la nappe d'Abidjan par un modèle de simulation des écoulements et $d u$ transport des polluants ». Thèse de Doctorat, Université d'Abobo Adjamé, Côte d'Ivoire, 212 p.

31. KOUADIO. G., DONGUI. B., TROKOUREY. A., (2000). «Détermination de la pollution chimique des eaux de la zone de la décharge d'Akouedo (Abidjan-Cote d'Ivoire) ». Revue des Sciences et Technologie, ENS-CI. Série A-01, 34-41.

32. KOLEDZI. E., TONYI AGBEBAVI. J., GNON. B., KOFFI. D., MATEJKA. G., (Mars 2015) ; "Gestion des déchets dans les villes en développement: transfert, adaptation du schéma et sources de financement » Article sur le journal scientifique Déchets Sciences et Techniques - N 68 - Novembre 2014, DOI: 10.4267/dechets-sciencestechniques.169. https://www.researchgate.net/publication/273950117. 
33. KOLEDZI. E. K., KPABOU. Y., BABA. G., TCHANGBEDJI. G., Kili. K. A., (2011a) ; «Composition of municipal solid waste and perspective of decentralized composting in the districts of Lome, Togo». Waste Management, 31 pp: 603 - 609.

34. KOLEDZI K.E., BABA. G., TCHANGBEDJI. G., AGBEKO. K., MATEJKA. G., FEUIL - LADE. G., BOWEN. J., (2011b) ; « Experimental study of urban waste composting and evaluation of its agricultural valorization in Lome (Togo) ». Asian J. Applied Sci., 4, pp : 378-391.

35. KOLEDZI. K. E., (2011) ; «Valorisation des déchets solides urbains dans les quartiers de Lomé (Togo) : approche méthodologique pour une production durable de compost $»$. Thèse de doctorat $\mathrm{N}^{\circ} 04-2011$ de l'Université de Limoges.

(http://epublications.unilim.fr/theses/index.php?id=7131).

36. MAQUERON. G., (25 Novembre 2009); «Déchets ménagers : composition de la poubelle », Dossier sur le recyclage et le traitement des déchets, Planète.

37. Ministère de l'Environnement et de l'Assainissement, Direction Nationale de l'Assainissement et du Contrôle des Pollutions et Nuisances (DNACPN) (novembre 2007), "Politique Nationale de l'Assainissement du Mali (PNA) », version provisoire.

38. MIZERO. M., NDIKUMANA. T., et JUNG. G. C., (20 décembre 2015) "Quantification, caractérisation et voies de valorisation des déchets solides municipaux dans la ville de Bujumbura»; Article publié au Bulletin Scientifique sur l'Environnement et la Biodiversité dans la discipline Sciences du vivant : Sciences de l'environnement \& écologie. Reference: http://hdl.handle.net/2268/220775. ISSN: 25190113, e-ISSN: 2519-0121.

39. MOKHTARIA. M. M., EDDINE. B. B., LARBI. D., AZZEDINE. H., RABAH. L., (2007) ; "Caractéristiques de la décharge publique de la ville de Tiaret et son impact sur la qualité des eaux souterraines », Courrier du savoir, $n^{\circ} 8$ : 93-99.OMS (1980). Nitrates, Nitrites et composés $\mathrm{N}$ - nitro so, Génève, $112 \mathrm{p}$.

40. Mairie de la Commune VI / Comité de Gestion et de Valorisation des Déchets (COGEVAD) (janvier 2006), "Ouvrages / équipements d'assainissement individuels et collectifs, en Commune VI du District de Bamako : données quantitatives ».

41. MUZUMBI M., (2008) ; "Assainissement urbain par l'approche « pollueur payeur » au quartier Matonge, dans la commune de Kalamu à Kinshasa». Mémoire de fin d'étude / Institut Facultaire de Développement (IFAD), 59 pages. 
42. NAQUIN. P., \& NGNIKAM. E., (2008). «Compostage des déchets ménagers dans les pays en développement : Modalités de mise en place et de suivi d'installations décentralisées pérennes ». Guide CEFREPADE, ADEME.

43. NAQUIN. P., \& al. (Mai 2008), «Projet pour la mise en place d'une plateforme expérimentale de compostage des déchets ménagers à Kasba Tadla (Maroc) », CEFREPAD - INSA de Lyon, Municipalité de Kasba Tadla et FST - BM. Lien du document : https://compostagecefrepade.files.wordpress.com/2009/01/projetcompostage-kasba-tadla-mai08.pdf

44. NAQUIN. P., \& GASTON. J., (2020), «Modalités de gestion du service public des déchets », PAGEDS ; Document répertorié sous le numéro A 196, sur la plateforme «Réseau pour la gestion durable des déchets», Documents et contact accessibles sur: www.amorce.org/decheteque.

45. NAQUIN. P., ZAHRANIA. F., NGNIKAMC. E., (March 2015); "Pré collecte des déchets ménagers dans les pays en développement: comment évaluer les actions menées ? »; Article publié dans la Revue Francophone d'Ecologie Industrielle - Trimestriel - $\mathrm{N}^{\circ} 43$ - Parution de Septembre 2006 - DOI: 10.4267/dechets-sciencestechniques.1868; https://www.researchgate.net/publication/279698159.

46. NGAHANE. E. L., (21 janvier 2015); "Gestion technique de l'environnement d'une ville (bembereke au bénin): Caractérisation et quantification des déchets solides émis; connaissance des ressources en eau et approche technique ». Thèse de doctorat en Sciences de l'environnement et écologie de l'Université de Liège, Liège, Belgique. Reference: http://hdl.handle.net/2268/177863.

47. NGO. C., SAMUEL. M., \& REGENT. A., (2004) ; «Déchets et pollution: Impact sur l'environnement et la santé ». France : Dunod, p 123 NGUYEN.

48. NKITUAHANGA YENAMAU. A., (2009); "Problématique de la gestion des ordures ménagères dans la ville de Kinshasa, cas de la commune de Masina ", mémoire pour l'obtention du diplôme Master en Ingénierie de l'Eau et de l'Environnement, Option: Environnement Université de Kinshasa.

49. NTABUGI KIKONGO. M. M., (2013); "Analyse du niveau de connaissances de la population de la ville de Bukavu sur la gestion des déchets ménagers : cas de la commune d'Ibanda, sud - kivu, République Démocratique du Congo »; Superviseur : Prof Dr. Jean Pierre BALUKU BAJOPE; Co-Superviseur: Prof Dr. Pascal 
ISUMBISHO MWAPU ; Université BIOSADEC, République Démocratique du Congo.

50. OJEDA - BENITZ S \& al. (2003), « Characterization and quantification of household solid waste in a Mexican city ». Resources, Conservation and recycling 39: 211 - 222.

51. ONIBOKUN et al. (2002), « La gouvernance et la gestion des déchets en Afrique ». CRDI. Canada, 11pages.

52. Organisation Néerlandaise de Développement, SNV - Mali, (août 2008), "Répertoire des GIE de pré collecte d'ordures ménagers intervenant dans le District de Bamako : cas de la commune VI», Etude diagnostique, Rapport provisoire. Adresse : Badalabougou - Est Rue 17 Porte 305, Téléphones : 002232233347 / 48 Fax : 00223 22310 84. Site - web: http://www.snvworld.org/en/countries/mali.

53. OTAYEK. R., (2009). "Décentralisation et nouveaux acteurs locaux ». Centre d'Etudes d'Afrique Noire.

54. OUSSEINI A., (2011), "Proposition de filières de recyclage des déchets plastiques de la ville de Conakry», Mémoire de maîtrise, Université GANC/IPC, Guinée, 106 pages.

55. 5SEGBEAYA K.N., (2012) ; «Evaluation de l'impact des déchets ménagers de la ville de Kara sur la qualité de la rivière Kara (Togo) ». Thèse de doctorat $N^{\circ} 399$ / FDS de l'Université de Lomé.

56. SEGBEAYA. N. K., BABA. G., FEUILLADE. G., DJAHINI. K., PALLIER. V., KOLEDZI. K. E., KILI. K., MATEJKA. G., (2012); "Caractérisation de la Matière Organique Dans les Matrices Déchets, Eaux et Sédiments Comme Indicateur D'évaluation de la Dégradation de la Qualité de la Rivière Kara Par les Déchets Solides Municipaux»; Article publié dans European Journal of Scientific Research.

Reference: http://www.europeanjournalofscientificresearch.com. ISSN 1450216X Vol.75 No.4 (2012), pp. 598-610 @ EuroJournals Publishing, Inc. 2012.

57. TCHEGUENI S., (2011) ; «Contribution à la valorisation des déchets agro-alimentaires en compost : Caractérisation physico - chimique des composts et étude de leur minéralisation dans deux sols agricoles $d u$ Togo ». Thèse de doctorat - $\mathrm{N}^{\circ} 363$ / FDS de l'Université de Lomé.

58. THUAL J.., 2016 Août (mise à jour) ; " gérer les déchets organiques sur un territoire »; ADEME Angers (Agence de l'Environnement et de la maitrise de l'Energie) ; 20 avenue du Grésillé, BP 90406 ; 49004 Angers Cedex 01.

Documents des plateformes Re - Sources et Amorce :

59. https://www.plateforme-re-sources.org/fiches-synthetiques/

60. https://www.plateforme-re-sources.org/guides/ 
61. Fiche technique de compostage : https://www.plateforme-resources.org/wp-content/ uploads/2015/05/FS-Compostage.pdf

62. Guide caractérisation : https://www.plateforme-re-sources.org/wpcontent/uploads/2018/07/ Guide-operationnel-caraterisation.pdf

63. Compostage des déchets ménagers dans les pays en développement : modalités de mise en place et de suivi d'installations décentralisées pérennes, 2012. Guide rédigé par les experts du CEFREPADE, document disponible sur :

64. https://www.pseau.org/outils/ouvrages/cefrepade_compostage_des_d echets_menagers_dans_les_

pays_en_developpement_modalites_de_mise_en_place_et_de_suivi_ d_installations_decentralisees_perennes_2012.pdf 